\title{
La formulación química en la formación inicial del profesorado: concepciones y propuestas
}

\author{
Manuel Fernández-González \\ Departamento de Didáctica de las Ciencias Experimentales.Facultad de Ciencias de la Educación. \\ Universidad de Granada.E-mail:mfgfaber@ugr.es.
}

[Recibido en febrero de 2013, aceptado en junio de 2013]

\begin{abstract}
La enseñanza de la formulación química, que se realiza en la segunda etapa de la ESO, constituye por sus dificultades un verdadero reto para el profesorado del que difícilmente sale airoso. El problema es delicado porque, además, puede generar actitudes de rechazo de los alumnos que muchas veces conducen a un abandono de los estudios de ciencias. El presente trabajo aborda el tema centrándose en el profesor y, concretamente, en el profesor en formación. Se han estudiado las concepciones que tiene acerca de cómo llevar a cabo la enseñanza del tema y se ha comprobado que responden a unos patrones que constituyen lo que podría llamarse un modelo docente espontáneo. Tras la crítica correspondiente, se ha diseñado una secuencia para el curso de formación que tiene en cuenta estas concepciones e incluye, además, una propuesta alternativa de enseñanza inicial de la formulación.
\end{abstract}

Palabras clave: enseñanza de las ciencias; formulación química; formación inicial del profesorado; preconcepciones sobre enseñanza.

Chemical formulation in the initial training of secondary school teachers: conceptions and proposals

Chemical formulation in Spain is taught in middle school. Because of its difficulty, it is a considerable challenge for secondary school teachers. This problem is a serious one because when chemical formulation is not well taught, something that happens all too frequently, students can acquire a negative attitude towards chemistry. This can subsequently lead them to reject the subject completely and even make them decide against studying science at all. Most research on this topic is centered on the student. However this study focuses on the teacher and the difficulties inherent in teaching chemical formulation. As such, it is particularly relevant for preservice secondary school teacher training. For this purpose, a study was made of teachers' conceptions of how formulation should be taught. The results obtained showed that these conceptions are in consonance with a set of tendencies and patterns that constitute a spontaneous teaching model. After a critical analysis of this model, a training course is presented that takes these tendencies and patterns into account. It includes a didactic proposal of how to teach chemical formulation more effectively.

Key words: science teaching; chemical formulation; preservice teacher training; preconceptions of teaching.

\section{Introducción}

La investigación didáctica comenzó hace varias décadas ocupada en dilucidar los errores e ideas que profesaba el alumno. Sus resultados permitieron conocer mejor las tendencias y concepciones que asumía, lo que hizo posible la puesta a punto de estrategias y modelos encaminados a hacer la enseñanza más efectiva. Pronto se reveló que el profesor no estaba a salvo de muchas de las deficiencias detectadas (Burgoon et al., 2011), lo que repercutía en su actividad docente, desarrollada además siguiendo una metodología poco adecuada. A partir de ahí la investigación didáctica se ha volcado cada vez más en la figura del profesor (Abell, 2007), adquiriendo gran importancia la formación del profesorado como componente imprescindible del sistema educativo. A este respecto el informe Rocard de la Comisión Europea (2008) es concluyente:

"... aunque los jóvenes poseen una curiosidad natural por estas materias, la educación cientifica formal tradicional puede abogar este interés y, por lo tanto, puede tener un impacto negativo sobre el desarrollo de actitudes hacia el aprendizaje de la ciencia [...] El profesorado es la piedra angular de cualquier 
renovación de la educación cientifica. Las aptitudes de los profesores (contenido y pedagogía)... son cruciales" (p. 108-110).

En consecuencia, hoy día la didáctica de las ciencias perfila las exigencias en la formación del profesorado (Carrascosa et al., 2008). Los estudios han centrado su atención en un elemento fundamental, presente en la mayor parte de las investigaciones, como es el conocimiento didáctico del contenido (CDC, o en inglés PCK), que es el conocimiento que debe tener el profesor sobre cómo enseñar unos contenidos determinados (Acevedo, 2009). El concepto ha adquirido gran importancia en las propuestas docentes, desplazando hacia sí la atención que antes recaía sobre el conocimiento del contenido (CC, o en inglés SMK). Su promotor, Shulman (1987), consideró inicialmente cinco categorías de conocimiento junto al CC y al CDC. Otros autores (p.ej. Zeidler, 2002) han señalado algunas menos, esto es, CC, CDC y CD (CD: conocimiento didáctico, en inglés PK). En esencia, como el CDC es la aplicación del CD a una temática particular, el esquema puede simplificarse en dos núcleos principales, el disciplinar y el didáctico. Habitualmente entre los futuros profesores nos encontramos el primero desarrollado y el segundo inexistente (Shulman, 1985), circunstancias poco halagüeñas porque la ausencia de formación didáctica repercute negativamente en el propio conocimiento científico que se transmite (Mellado y González, 2000). De todos modos, es factible promover estrategias para superar una situación como esta, en la que, sólo con el CC adquirido en los estudios previos, no pueden tomarse decisiones adecuadas sobre qué enseñar y cómo hacerlo (Roehrig et al., 2007).

Por otra parte, contemplando la situación desde el punto de vista del contenido, no es raro encontrar en cada disciplina algún tema tan esencial que se considera la llave de acceso. Es el caso de la formulación química, que aporta el "lenguaje de la química" y constituye una herramienta básica, tanto descriptiva como heurística (Taskin y Bernholt, 2012). La fórmula química va a ser la protagonista de nuestra investigación. A nivel macroscópico representa una sustancia. A esto añade información sobre su composición cualitativa (elementos componentes) y cuantitativa (proporción entre ellos). A nivel microscópico señala la proporción entre sus átomos y, si es el caso, representa además el número de átomos de cada elemento que compone su molécula (Gabel, 1998).

En el estudio de la formulación el peligro es que se atienda más al símbolo que a lo simbolizado y, sobre todo, que, en aras a su importancia, pueda desencadenar una enseñanza desmedida. La precaución debería ser extrema en los cursos de iniciación, esto es, $3^{\circ}-4^{\circ}$ de ESO. Pero en la práctica no ocurre así, y los resultados de enseñanza suelen ser habitualmente poco satisfactorios. A este respecto, conviene tener presente que el primer contacto con la formulación química resulta siempre decisivo para los alumnos. Una enseñanza inadecuada puede acarrear consecuencias particularmente graves, pues provoca actitudes negativas hacia la asignatura y, por extensión, hacia la ciencia en general (Vázquez y Manassero, 2008). Todos conocemos casos de abandono de los estudios de ciencias por estos motivos.

En el presente trabajo pretendemos acometer el problema desde su raíz y para ello vamos a centrarnos en la formación inicial del profesorado. Comenzaremos entonces estudiando las ideas que los futuros docentes profesan acerca de la formulación química y la manera de enseñarla. Estas concepciones serán analizadas para poner de manifiesto sus características y tendencias. Siguiendo los resultados obtenidos, se acometerá la tarea de diseñar un curso de formación que incluirá una propuesta alternativa de enseñar formulación a nivel inicial. La propuesta cuidará de que sea una enseñanza razonada, lejos del operativismo mecánico habitual. 


\section{Metodología}

Se ha seguido una secuencia de corte constructivista, experimentada durante varios años en cursos de formación inicial de profesores. La muestra estudiada ha sido recogida tanto del Máster de Secundaria, como del CAP, y de la asignatura "Didáctica de la Física y la Química" seguida por alumnos de $4^{\circ}-5^{\circ}$ de Licenciatura. Todos estos cursos tienen en común sus esfuerzos hacia la formación didáctica de los futuros profesores de secundaria. Puesto que nuestro estudio está dirigido a averiguar tendencias generales sin descender a ningún tipo de análisis fino, no se han hecho distinciones entre ellos.

El trabajo se ha desarrollado en tres etapas. En primer lugar se realiza una prueba diagnóstica para poner de manifiesto las creencias de los futuros profesores acerca de cómo debe enseñarse la formulación. La prueba permitirá mostrar concepciones relacionadas con la formulación, que son las que determinan la estrategia de actuación docente prevista. El origen de tales concepciones puede residir en una escasa capacidad de adaptación al nivel inicial de los alumnos, en la reproducción de hábitos y estrategias de enseñanza recibidos (Valcárcel y Sánchez, 2000), y en las propuestas de los libros de texto.

A continuación se va a llevar a cabo un estudio crítico de cada una de estas concepciones, señalando su mayor o menor idoneidad, y considerando igualmente el efecto conjunto de todas ellas. De aquí podrán deducirse las líneas directrices del esquema previsto de actuación docente, compartido por la mayoría de los futuros profesores. Presumiblemente será también el asumido por muchos profesores en ejercicio.

Pasamos entonces a la segunda etapa, equivalente a la misma de Driver (1986), donde se ponen en cuestión las ideas más improcedentes aparecidas. Se trata de ir erosionándolas con el objetivo de reconducir la situación. Esta etapa corresponde a la primera parte del curso de formación propiamente dicho. Se comienza pues enfrentando a los futuros profesores a varias situaciones problemáticas reales (Singer et al., 2011), relacionadas con las ideas anteriores, que deberán estudiar y elaborar luego unas conclusiones. Esta etapa ha de ser complementada con un análisis por parte del profesor coordinador de los comentarios emitidos, así como su juicio crítico acerca de la situación, señalando las causas de la inadecuación a la enseñanza de ciertas concepciones.

Preparado entonces el terreno, puede ya pasarse a la tercera etapa (segunda del curso), en la que se introduce una nueva propuesta de enseñanza de la formulación química. Como tiene en cuenta la inadecuación de las concepciones habituales, evita sus inconvenientes y ofrece, en su lugar, otras de interés didáctico.

\section{Prueba diagnóstica. Resultados}

El propósito de la prueba inicial era explicitar las ideas de los futuros profesores acerca de qué enseñar y cómo enseñar sobre la formulación química a nivel inicial. La prueba consistía en un trabajo, propuesto para hacer en casa, sobre la siguiente cuestión (A0):

A0. Hacer un diseño didáctico para explicar la formulación química (inorgánica) a nivel inicial $\left(3^{\circ}-4^{\circ}\right.$ de ESO). Especificar los contenidos mínimos que se impartirían y la manera de enseñarlos. Comentar con detalle el desarrollo de la propuesta.

La prueba es totalmente abierta, siendo su única limitación una extensión mínima fijada en tres páginas. Esta libertad de acometer el desarrollo lleva consigo que no todos los comentarios recogidos inciden en los mismos tópicos. Algunos tópicos pueden haber sido ignorados, otros 
expuestos muy brevemente y otros sólo citados sin más explicaciones. Por ello el número de respuestas a cada uno no coincide con el número total de encuestados.

Se han recogido las respuestas de 49 futuros profesores matriculados durante estos últimos años (2008-12) en los cursos de formación antes citados. Las respuestas están referidas fundamentalmente a cuatro bloques temáticos (R1-R4), que se han desglosado en apartados. La Tabla 1 muestra resumidamente los diversos tópicos y el número de profesores en formación que los suscribe.

Tabla 1. Tópicos tratados por los futuros profesores.

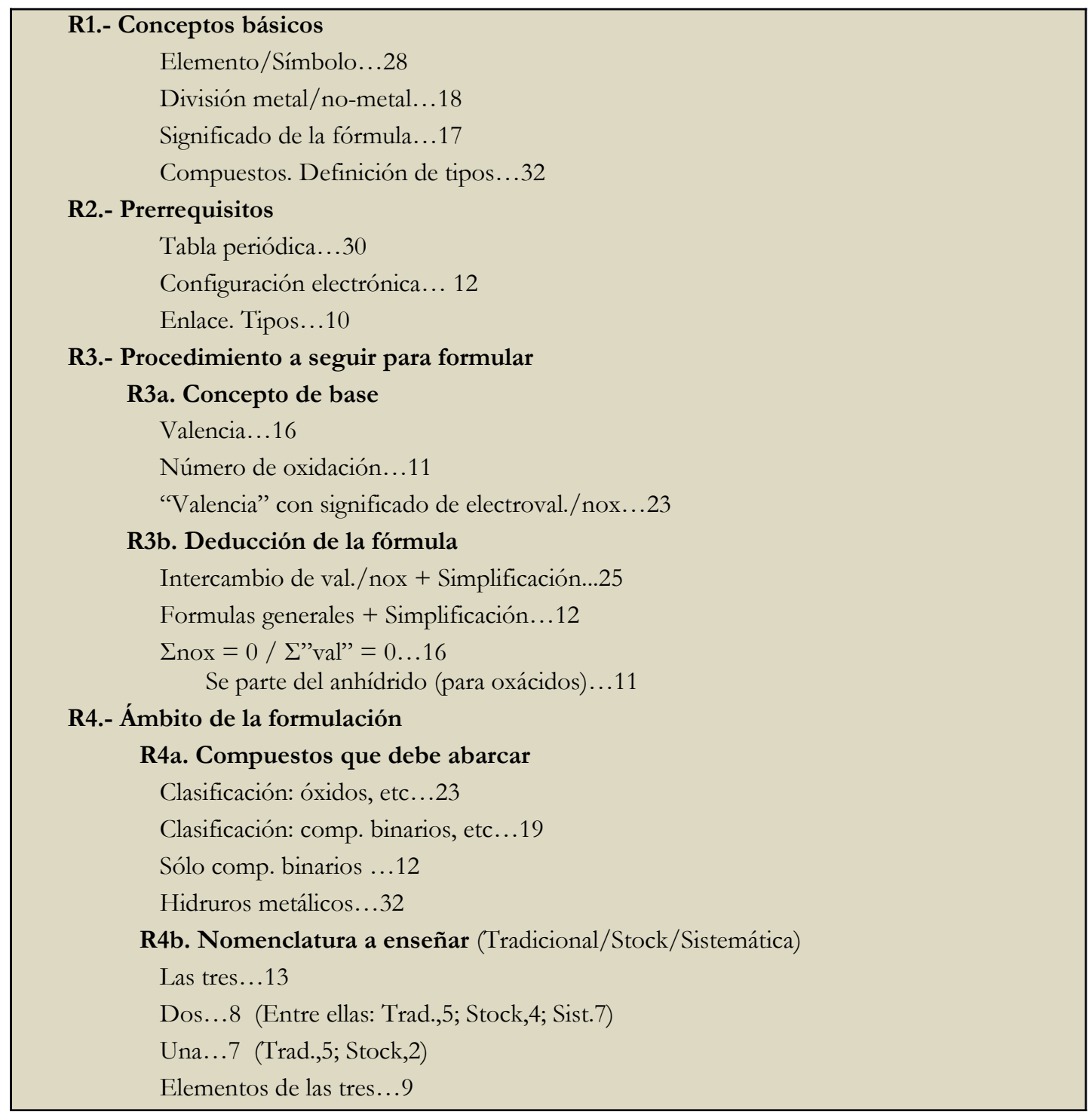

Conviene aclarar que, respecto al procedimiento de deducción de la fórmula (R3b), a veces se sugiere el uso de fórmulas generales (p.ej. $\mathrm{M}_{2} \mathrm{O}_{\mathrm{n}}$ para el óxido de un metal de valencia/nox $n$ ). En cuanto al ámbito que debe abarcar inicialmente la formulación, se incluyen clasificaciones de compuestos (R4a) porque fuerzan siempre la ampliación del ámbito. Es de señalar, además, que entre los tipos de sustancias, las simples (p.ej. $\mathrm{H}_{2}$ ) aparecen escasamente (10, que no se han incluido en la tabla). 


\section{Análisis de resultados}

Vamos ahora a analizar y comentar las respuestas (R) de la prueba diagnóstica, recogidas en la Tabla 1 y siguiendo sus diversos apartados.

\section{R1. Conceptos básicos}

Es obvio empezar la formulación recordando qué son los elementos, sus símbolos y la división metales/no metales. También es de interés enseñar en qué consiste cada tipo de compuesto (óxido, etc.) antes de abordar la formulación de cada uno. Es esencial igualmente conocer el significado de una fórmula, aunque sobre esta cuestión no se insiste demasiado en las propuestas. Tampoco se menciona, a pesar de su interés, la distinción entre los conceptos de formulación y nomenclatura.

\section{R2. Prerrequisitos}

Se considera mayoritariamente prerrequisito para la formulación el conocimiento de la tabla periódica, bien en su totalidad (sin los elementos $4 f$ y $5 f$ ), bien una parte sustancial de la misma. La razón señalada es que se contempla como fuente de donde emanan todos los ingredientes que van a utilizarse: elementos, símbolos, división metales/no metales, familias, configuraciones electrónicas, electronegatividades y números de oxidación.

Otro prerrequisito, relacionado con el anterior y al que se alude con frecuencia, es la necesidad de conocer las configuraciones electrónicas de los elementos. El motivo reside, tal y como se explicita en algunas exposiciones, en que de ese modo "las valencias no tienen que aprenderse de memoria". Igualmente, otros creen que resulta útil para comprender el enlace, aunque el papel de este en la formulación no aparece nunca claro.

\section{R3. Procedimiento a seguir para formular}

R3a. Suele basarse o en la valencia del elemento o en su número de oxidación (nox). Recordemos que la valencia se define como el número de enlaces que un átomo del elemento forma al unirse con otros. Viene dada por un simple número. El nox es un concepto mucho más abstracto, y se entiende como la carga que tendría un átomo en un compuesto si los electrones de los enlaces se asignasen al átomo más electronegativo. Viene dado por un número precedido de un signo. Un error muy común es hacer sinónimos estos dos conceptos, que en muchas ocasiones coinciden numéricamente (Smith, 2005). Es de señalar, además, el empleo mayoritario de un concepto ambiguo, híbrido entre ambos, designado como "valencia", pero unido a un signo. Este concepto de "valencia" no siempre coincide con el de nox (p.ej. $\mathrm{H}_{2} \mathrm{O}_{2}$ : "v" $(\mathrm{O})=-2 ; \operatorname{nox}(\mathrm{O})=-1$ ).

R3b. En los compuestos binarios el procedimiento más ampliamente seguido para obtener la fórmula consiste en aplicar la "regla del intercambio", sea este de valencias o de nox, seguida en muchos casos por la "regla de simplificar" (dividiendo por 2 normalmente). Otra modalidad más minoritaria está basada en las fórmulas-tipo generales (que hay que aprender), que aunque aporta la constitución del compuesto, para los binarios el método en esencia se reduce al anterior. En el caso de oxácidos (y oxisales) se suele utilizar un procedimiento de formulación constructiva que conduce a la fórmula partiendo del anhídrido (o de sus elementos), "sumando" agua y, con frecuencia, "dividiendo" por 2. Los hidróxidos se tratan como compuestos binarios, con el grupo/ion hidróxido actuando como una sola entidad. Otra propuesta recogida, que vale para todos los compuestos, parte de los nox (o las "valencias") y aplica la norma de "compensar las cargas", añadiendo átomos de alguno de sus elementos para que la suma algebraica de los nox (o las "valencias") del compuesto sea cero. 


\section{R4. Ámbito de la formulación}

R4a. Las sustancias que aparecen suelen encontrarse clasificadas según dos criterios principales, aunque no es raro encontrar ambos superpuestos. Una clasificación utiliza el criterio de tipos funcionales (óxidos, etc.) y la otra, suscrita casi por igual, se basa en el número de elementos (compuestos binarios, etc.). El uso sobrevalorado de la clasificación impregna gran parte de las propuestas e impulsa a ampliar los límites de los contenidos a enseñar. En consecuencia, se da cabida junto a óxidos, hidróxidos, ácidos y sales, a compuestos de menor importancia como hidruros metálicos, peróxidos y sales básicas. Otras propuestas en cambio, siguiendo una desafortunada directriz ministerial, estiman que la formulación inicial debe abarcar sólo a los compuestos binarios (MEC, 2007: 699, "Bloque 4"). Paradójicamente el tratamiento que hacen de estos es exhaustivo e incluyen todos sus tipos posibles. La fuerza de la clasificación es tal que cuando se habla de hidruros no metálicos (ácidos hidrácidos) se crea la obligación de hablar también de hidruros metálicos. De ahí que, entre unas cosas y otras, la enseñanza de estos rara vez falta en las propuestas.

R4b. Respecto a la cuestión acerca de qué tipo de nomenclatura debe ser enseñada, la opinión más extendida es que hay que enseñar las tres: la tradicional, la de Stock y la sistemática. Otros encuestados, en cambio, no se definen por ninguna y utilizan normas de unas y de otras. Y para los que sólo citan una, la preferida es la tradicional.

\section{Modelo docente espontáneo}

Los resultados obtenidos en la prueba diagnóstica, que acaban de analizarse, han de ser tomados como indicios que revelan causas más profundas. Estas hay que buscarlas en las creencias del profesor novel sobre el enfoque a dar a su actuación docente. Entre ellas encontramos algunas de tipo general, otras son estructurales, y también las hay referidas a conceptos o procedimientos. De modo sucinto estas concepciones (C) son:

\section{C1. Perfil disciplinar}

En cuanto a las concepciones generales debe señalarse especialmente el perfil disciplinar de la enseñanza que se propone, cuyos rasgos principales son: un nivel conceptual elevado (p.ej. uso del concepto de nox) y un exceso de contenidos (p.ej. uso de los tres tipos de nomenclatura).

\section{C2. Afán clasificatorio}

Esta característica estructural encuentra aquí un terreno muy propicio. El afán clasificatorio es una tendencia a aplicar desmesuradamente este recurso. Tiene como consecuencia hacer obligatorio incluir compuestos de menor importancia (p.ej. hidruros metálicos) en aras a no dejar ningún hueco en la clasificación. El tema de la clasificación arrastra también la cuestión de elegir para ella un criterio más teórico (p.ej. compuestos binarios, etc.) u otro más tradicional por tipos funcionales (óxidos, etc.).

\section{C3. Concepciones particulares}

Son más concretas y propias de la temática. Entre ellas encontramos dos masivamente suscritas en las propuestas:

C3a. Tópicos elevados al rango de prerrequisito imprescindible para iniciarse en la formulación, como por ejemplo la tabla periódica,

C3b. Contenidos procedimentales discutibles, como por ejemplo la "regla del intercambio". 
Salta a la vista que estas características y tendencias están lejos de ser idóneas (ver ap. siguiente) y pueden acarrear consecuencias de enseñanza no deseables. Será, pues, preciso tenerlas en cuenta y esforzarse en corregir. Por otra parte, no es difícil percibir la implicación de unas con otras (p.ej. los excesos en el uso de la tabla periódica, C3a, contribuyen a acentuar el perfil disciplinar, C1).

¿Podemos decir entonces que el conjunto anterior de concepciones constituye un modelo docente espontáneo del profesor inicial? Como los rasgos puestos en evidencia, tomados de la realidad escolar, caracterizan una actuación docente prototípica y están conectados entre sí formando un esquema relacional, sí puede hablarse de un modelo de actuación. ¿Espontáneo? También, porque es la respuesta del futuro profesor a una situación nueva que nadie, por el momento, le ha enseñado a abordar.

Si algo caracteriza al modelo docente espontáneo es la carencia de conocimiento didáctico del contenido (CDC), ya que no recurre a ninguna norma avalada por la investigación didáctica. En tales circunstancias sus únicas referencias son los conocimientos (disciplinares) adquiridos en sus estudios y el ejemplo (normalmente tradicional) recogido de los profesores que ha tenido (López-Gay, 2012). Así pues, la tarea que se presenta a los responsables de los cursos de formación consiste esencialmente en sustituir el modelo docente espontáneo por un modelo didáctico fundamentado. Esto implica desarrollar el CDC y, por tanto, poner en práctica un cambio metodológico que sirva, además, de guía a una transposición didáctica (Chevallard, 1997).

\section{Crítica del modelo}

Antes hemos avanzado lo inadecuado de las concepciones que constituyen el modelo docente espontáneo. Vamos a justificarlo ahora con más detalle.

\section{C1. Perfil disciplinar}

Puesto que se trata de iniciar la formulación química y estamos situados en el $2^{\circ}$ ciclo de la ESO (alumnos de 14-15 años), el perfil disciplinar que se propone es, a todas luces, improcedente. Basta constatar el uso de nociones muy teóricas como la de nox, o de tópicos como las configuraciones electrónicas. Pero lo peor, quizás, es el número de conceptos de índole diversa que son transmitidos simultáneamente. El alumno se ve arrollado por una conjunción de todos ellos que le hacen imposible su correcta asimilación.

\section{C2. Afán clasificatorio}

El perfil disciplinar se encuentra reforzado por el afán clasificatorio, el cual termina introduciendo compuestos como los hidruros metálicos, a igualdad de trato con óxidos o sales. En cuanto a las modalidades de clasificación, es preferible una por funciones más próxima a la realidad (óxidos, etc.), que otra teórica y analítica (compuestos binarios, etc.). Esta última hace aparecer en diferentes categorías compuestos que tienen propiedades generales comunes (p.ej. las sales), lo que no ocurre con la primera, que los muestra agrupados.

\section{C3a. Concepciones particulares: la tabla periódica}

Es muy común la creencia en el papel primordial de la tabla periódica para saber formular. Es cierto que, entre otras cosas, puede facilitar la retención de valencias/nox y la asimilación de los tipos de compuestos, según los distintos grupos. Pero ello a costa de un esfuerzo memorístico más profundo. No debe olvidarse que siendo la tabla periódica una ordenación 
global, incita a considerar más elementos químicos de los necesarios para una enseñanza inicial de la formulación. Dejemos para los escolares que hayan elegido la rama de ciencias en una etapa posterior, estudiar la tabla periódica como apoyo a lo aprendido, y las configuraciones electrónicas como base de la tabla.

\section{C3b. Concepciones particulares: las reglas del intercambio y la divisibilidad}

En cuanto al procedimiento utilizado para formular, las reglas "del intercambio" y "la divisibilidad" acarrean inconvenientes nada despreciables. Tal y como se enseñan, aparecen ante el alumno como reglas aritméticas que se aplican mecánicamente, sin mostrar su fundamento químico (esto es, no puede quedar un elemento con valencias libres o un compuesto con la suma de nox diferente a cero). Para colmo de males, en numerosos casos (p.ej. Ca y O) se aplica la primera regla (que daría $\mathrm{Ca}_{2} \mathrm{O}_{2}$ ) y a continuación hay que modificar el resultado dividiendo por 2 (lo que da $\mathrm{CaO}$ ). Todo ello suena a arbitrario, y aún más considerando las excepciones que hay que tener en cuenta (p.ej. sólo entre los compuestos inorgánicos tenemos: $\mathrm{H}_{2} \mathrm{O}_{2}, \mathrm{H}_{2} \mathrm{~S}_{2} \mathrm{O}_{8}, \mathrm{Hg}_{2} \mathrm{Cl}_{2}$, etc.). $\mathrm{La}$ "regla de la divisibilidad" (" $\mathrm{Ca}_{2} \mathrm{O}_{2}=$ CaO") atenta, además, contra uno de los principios más esenciales de la química: si la composición de dos moléculas es diferente (aunque sea por un solo átomo), estas pertenecen a dos compuestos distintos. Es más, como sabemos al estudiar algunos tipos de isomería, basta que la estructura sea diferente para que los compuestos también lo sean.

\section{En conclusión}

La primera de las concepciones citadas (C1) es posiblemente aquella sobre la que más hay que trabajar en los cursos de formación inicial. El no saber (ni querer en algunos casos) adaptarse a la audiencia es típico de los profesores iniciales. A este respecto, es deseable potenciar destrezas en la selección de contenidos a fin de poder reducir de manera racional el exceso de los mismos. Esta tarea pasaría por empezar jerarquizándolos según su importancia.

En esencia, lo más preocupante del modelo docente espontáneo de formulación es el número de ingredientes conceptuales (algunos de alto nivel) transmitidos al mismo tiempo a los alumnos. Asimilar todo en conjunto es muy difícil y para muchos, imposible. Lo que debería ser - y permítaseme la metáfora culinaria - una pequeña taza de caldo, se convierte en una gigantesca olla de cocido... con tocino, morcilla y chorizo incluidos. La indigestión está servida.

\section{Primera fase del curso: reconducción de la situación}

Un curso de formación inicial del profesorado siguiendo las directrices apuntadas puede adoptar formas muy diversas. Aquí, en concreto, vamos a exponer el esquema que hemos puesto en práctica durante varios años, constituido fundamentalmente por dos fases: una de reconducción de la situación y otra de presentación de una propuesta alternativa.

La fase de reconducción con que se abre el curso ha de ir necesariamente encaminada a reflexionar sobre la validez didáctica de los componentes del modelo espontáneo. Pero también hay que señalar que la citada reflexión, siendo necesaria, no es suficiente si no se concreta en la práctica docente (López-Gay, 2012).

En esta primera fase, que sería equivalente a la "puesta en cuestión de las ideas previas" de Driver, se plantean varias cuestiones dirigidas a poner en evidencia alguno de los rasgos del modelo espontáneo. Se ha procurado adoptar para ellas la forma de situaciones de la vida cotidiana de clase ("casos problemáticos"). Los futuros profesores han de responderlas por escrito y, tras la revisión correspondiente, se discuten las opiniones vertidas. 
Mostramos cuatro actividades (A1-A4) que encierran intencionalidades concretas. A1 va dirigida a erosionar la concepción disciplinar (C1); A2 comparte esta finalidad y añade, además, una movilización contra los excesos clasificatorios $(\mathrm{C} 1+\mathrm{C} 2)$; A3 hace reflexionar sobre la atribución de protagonismo ilimitado a la tabla periódica (C3a); y A4 pone de relieve los problemas que acarrea la "regla del intercambio" (C3b).

A1. En esta primera actividad aparecen juntos un fragmento de partitura musical y otro de manual con fórmulas químicas. Se plantea entonces una cuestión que insinúa la similitud de ambas, ya que son representaciones que utilizan, una y otra, un lenguaje simbólico, con sus símbolos y reglas propios (Griffiths y Preston, 1992). Se incide también en el desconcierto de los no iniciados ante un lenguaje simbólico extraño.

\footnotetext{
A1. La figura muestra una partitura musical y ecuaciones y fórmulas químicas tomadas de un manual. a) ¿Por qué un estudiante de ciencias no comprende las primeras y un estudiante de conservatorio, las segundas? b) Pese a su diferente apariencia ¿tienen en común alguna característica esencial?
}

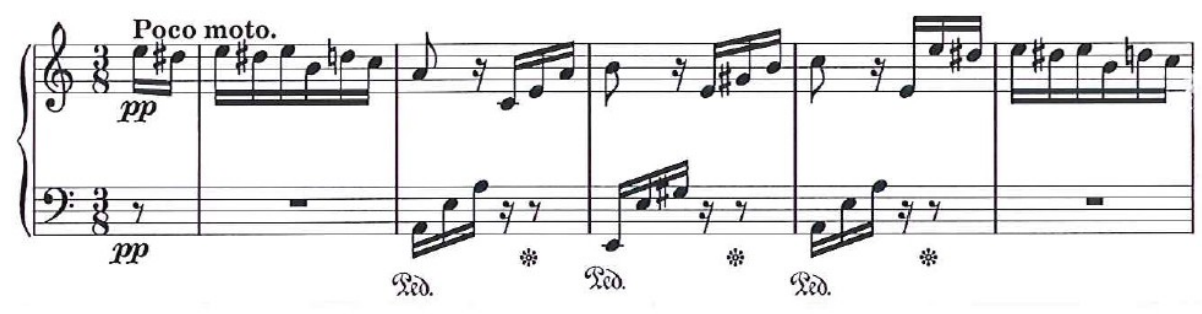

Reacciones de sustitución simple. En ellas un elemento desplaza a otro de un compuesto. Por ejemplo:

$$
\begin{aligned}
& \mathrm{Zn}(\mathrm{s})+\mathrm{H}_{2} \mathrm{SO}_{4}(\mathrm{aq}) \rightarrow \mathrm{ZnSO}_{4}(\mathrm{aq})+\mathrm{H}_{2}(\mathrm{~g}) \\
& \mathrm{Zn}(\mathrm{s})+\mathrm{CuSO}_{4}(\mathrm{aq}) \rightarrow \mathrm{ZnSO}_{4}(\mathrm{aq})+\mathrm{Cu}(\mathrm{s})
\end{aligned}
$$

Reacciones de doble sustitución. En ellas dos elementos se desplazan mutuamente de sendos compuestos. Por ejemplo:

$$
\begin{aligned}
\mathrm{AgNO}_{3}+\mathrm{NaCl} & \rightarrow \mathrm{NaNO}_{3}+\mathrm{AgCl} \\
\mathrm{CaCl}_{2}+\mathrm{Na}_{2} \mathrm{CO}_{3} & \rightarrow \mathrm{CaCO}_{3}+2 \mathrm{NaCl}
\end{aligned}
$$

A2. A continuación se propone un caso problemático en relación con la concepción disciplinar y exhaustiva de la enseñanza de la formulación.

A2. Un alumno de Magisterio tiene un examen en el que exigen formulación química a nivel elemental. Unos días antes del examen acude muy preocupado al profesor y le dice: "Todo iba bien hasta que pedí ayuda a un compañero de Quimicas. A partir de abi ya no entiendo nada".

¿Qué comentarios podríamos hacer de la situación?

A3. En el caso problemático que se da a continuación se aborda el problema de la sobrevaloración de la tabla periódica.

A3. Un profesor manifiesta que él pide a sus alumnos de $4^{\circ}$ de ESO que se aprendan la tabla periódica (TP) completa (excepto lantánidos y actínidos). Señala que, aunque invierte más tiempo, consigue unos buenos resultados: las tres cuartas partes de sus alumnos se la saben (demostrado en examen). Argumenta que "la TP es central en el estudio de la química y, por tanto, es preciso abordar su estudio pronto".

a) ¿Qué opinión le merece esta situación? Enumerar ventajas e inconvenientes.

b) ¿Qué haría si el profesor fuera Vd.?

A4. Por último se ofrece el caso problemático siguiente sobre la "regla del intercambio", complementada por la "regla de la divisibilidad". 
A4. Un profesor de Química enseña a formular a sus alumnos de Secundaria siguiendo un método según el cual "se intercambian las valencias y luego, si hay divisibilidad, se divide por 2". Pone el ejemplo del carbono y el oxígeno, escribiendo en la pizarra:

$$
\text { " } \mathrm{C}_{2} \mathrm{O}_{4}=\mathrm{CO}_{2} \text { " }
$$

En las siguientes clases comienzan a surgir algunas preguntas:

"Profesor ¿y el agua oxigenada $\left(\mathrm{H}_{2} \mathrm{O}_{2}\right)$ por qué no se divide por 2?"

(Otro alumno) "Profesor zy el butano $\left(\mathrm{C}_{4} \mathrm{H}_{10}\right)$ ?"

a) ¿Qué opinión le merece el método empleado?

b) ¿Y la actuación del profesor?

\section{Principios básicos de la propuesta}

Las actividades anteriores mueven, pues, al futuro profesor a iniciar una reflexión sobre las concepciones del modelo que suscribe. Y si, como es deseable, termina cuestionándolas, se mostrará más receptivo a asumir una nueva línea de actuación.

La propuesta alternativa que hemos diseñado para la formulación inicial descansa en varios principios:

1) No es didácticamente correcto enseñar al mismo tiempo contenidos poco asequibles. En el caso de la formulación sólo debe tener cabida la sola formulación. Hay que desbrozar el terreno de tópicos y conceptos colaterales como tabla periódica, configuraciones y enlaces (lo que no quiere decir que no vayan a estudiarse en otro momento). La excusa de la imposibilidad de desligar unos conceptos de otros no se sostiene. Es posible hacerlo siguiendo la estrategia y el nivel oportunos.

2) Se intentará un mayor acercamiento a la realidad. Por ejemplo, respecto a la clasificación, nos inclinamos por el criterio basado en tipos funcionales (óxidos, etc.), con preferencia al de base analítica (compuestos binarios, etc.). El primero se muestra más próximo al mundo real pues agrupa compuestos con propiedades generales comunes, con independencia de que estén formados por dos o tres elementos (p.ej. ácidos o sales). El mismo principio vamos a seguir para elegir la nomenclatura (punto 6).

3) La práctica de la formulación es, en esencia, establecer relaciones entre elementos. Por tal motivo proponemos hacer hincapié en el término relación y atenuar el término elemento, utilizando sólo unos pocos de ellos. En consonancia, van a desaparecer los requerimientos a la tabla periódica como guía de la formulación.

4) Va a erigirse el concepto clásico de valencia como concepto clave en la enseñanza de la formulación. De cada elemento seleccionado bastará saber su valencia, sin descender a explicaciones derivadas de la teoría electrónica. Con ello se justifican por innecesarias las exclusiones del punto 1. Tampoco se requiere el concepto de ion, y en su lugar se va a hablar de grupo con valencia propia (p.ej. "el grupo sulfato tiene dos valencias: $=\mathrm{SO}_{4}$ "). Así puede prescindirse del concepto de nox, que, además de abstracto, tiene sus limitaciones (Woolf, 1988). Podría objetarse que la aplicación del concepto de valencia supone que todos los compuestos son covalentes. Pero también el concepto de nox equivale a suponer que son, en cambio, iónicos. De todos modos, es posible una formulación inicial basada en el nox, memorizando los nox de los elementos (con la ayuda de la tabla periódica) o las reglas generales de asignación. A esto hay que añadir la dificultad de aprender el concepto, excesivamente teórico, que, además, arrastra a otros del mismo rango como el de electronegatividad.

5) Las reglas de formulación habituales ("intercambio" y "divisibilidad") van a ser sustituidas por otra basada en las valencias, que señala que los átomos se unen según estas, sin que pueda quedar ninguna libre en el compuesto. 
6) En cuanto a la nomenclatura, la idea-guía es acercarse en lo posible al nombre más comúnmente utilizado en la vida cotidiana y en el laboratorio. No emplearemos emplearemos, salvo alguna excepción, la nomenclatura sistemática. En consecuencia, $\mathrm{H}_{2} \mathrm{SO}_{4}$ será ácido sulfúrico y no tetraoxosulfato de dihidrógeno (!). También es rechazable enseñar los tres nombres del compuesto (Hernández y Palacín, 1993). No vamos, por tanto, a encasillarnos en ningún tipo estándar de nomenclatura sino que utilizaremos las reglas de unas u otras, según el compuesto.

Los principios anteriores constituyen las ideas directrices que han intervenido en la elaboración de la propuesta alternativa. Hay que reconocer que la propuesta no está exenta de memorismo - ello sería imposible - aunque este se encuentra reducido a su mínima expresión. Permite por el contrario, como vamos a ver, un aprendizaje razonado, que se fundamenta en el concepto de valencia y en los tipos de compuestos.

\section{Segunda fase del curso: propuesta didáctica}

Exponemos ahora con detalle la propuesta didáctica alternativa, señalando los cambios producidos en los bloques temáticos en relación a los resultados $(\mathrm{R})$ de la prueba diagnóstica.

\section{R1. Conceptos básicos.}

Los señalados en un principio (elemento, fórmula, tipos de compuestos) son adecuados y deben mantenerse. A estos hay que añadir la distinción entre los términos "formulación" y "nomenclatura". Es, sobre todo, especialmente importante conocer el exacto significado de una fórmula. Para explicarlo con claridad lo ideal es disponer de algún modelo molecular (bolas y varillas), como por ejemplo el de la molécula de agua (Wu, Krajcik y Soloway, 2001). Aunque los alumnos saben que la fórmula del agua es $\mathrm{H}_{2} \mathrm{O}$, su significado suele desconocerse o es erróneo. Con frecuencia el error reside en la confusión de los términos átomo y molécula ("el agua, $\mathrm{H}_{2} \mathrm{O}$, está formada de dos moléculas de hidrógeno y una de oxígeno"). Es necesario pues, siempre a la vista del modelo, clarificar esta cuestión, así como lo que representa el subíndice.

\section{R2. Prerrequisitos.}

Hemos reducido drásticamente el número de elementos químicos que van a emplearse (doce en total). Así el alumno centra su atención sólo en unos pocos, de los que tiene que aprenderse de memoria (si no lo sabe ya) sus símbolos y sus valencias. La selección ha sido realizada sin las servidumbres que impone la tabla periódica, más atenta al grupo que al elemento individual. El concepto de valencia se introduce, como se ha dicho, en su acepción clásica. Para nuestros propósitos basta aprender las valencias de ocho de los doce elementos seleccionados, especialmente metales (Ver Esquema 1).

\section{Esquema 1}

\section{ELEMENTOS SELECCIONADOS. SÍMBOLOS}

No metales: Hidrógeno, $\mathrm{H}$ / Oxígeno, O / Carbono, C / Cloro, Cl / Azufre, S / Nitrógeno, N

Metales: Sodio, Na / Potasio, K / Cinc, Zn / Calcio, Ca / Plata, Ag / Hierro, Fe

\section{VALENCIAS DE ALGUNOS DE ELLOS}

No metales: H: I / O: II / C: IV (II) [(II) significa que es poco frecuente en el C]

Metales: Na: I / Zn: II / Ca: II / Ag: I / Fe: II y III [Fe es ej. de valencia múltiple] 


\section{R3. Procedimiento a seguir para formular.}

Para empezar se proponen las siguientes cuestiones (A5-A6):

A5. ¿Por qué la fórmula del agua es $\mathrm{H}_{2} \mathrm{O}$ y no $\mathrm{HO}_{2}$ o $\mathrm{HO}$ ?

A6. ¿Por qué el $\mathrm{C}$ se une con el $\mathrm{O}$ formando un compuesto de fórmula $\mathrm{CO}_{2}$ ?

A5. Esta primera cuestión permite poner en práctica el procedimiento que vamos a seguir. Básicamente consiste en unir las valencias de los átomos y formar "enlaces", cuidando de que no queden valencias libres. Si esto no ocurre a la primera, se añade algún átomo más que aporte valencias que saturen las que quedaron libres (Esquema 2).

Esquema 2

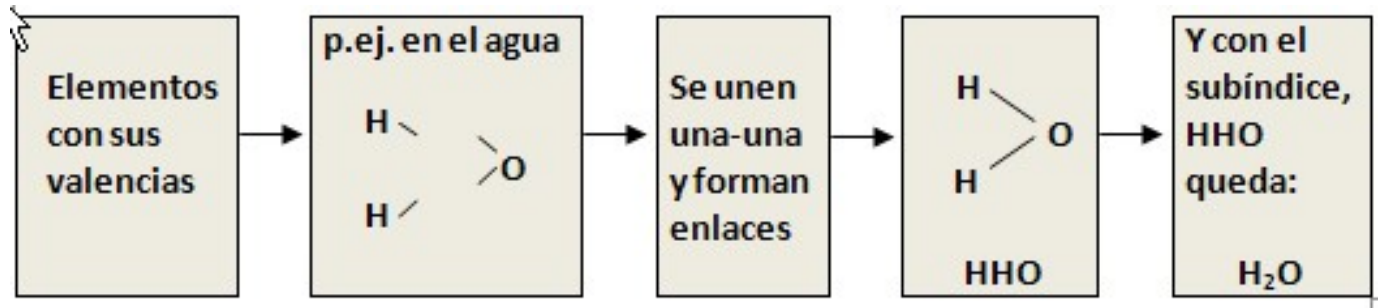

A6. El desarrollo de la segunda cuestión pone de manifiesto lo innecesario de las reglas del "intercambio" y del "simplificar". Aplicando el procedimiento anterior se despliegan las cuatro valencias del carbono, se enlazan con las dos de un oxígeno y se añade otro para que no queden valencias libres (Esquema 3). El resultado directo: $\mathrm{CO}_{2}$. Con esto se evitan explicaciones desafortunadas del estilo de: “... resulta $\mathrm{C}_{2} \mathrm{O}_{4}$, que es lo mismo que $\mathrm{CO}_{2}$ ” (!).

Esquema 3

$$
\mathrm{O}=\mathrm{=}=\rightarrow \mathrm{O}=\mathrm{C}==\mathrm{O} \rightarrow \mathrm{O}=\mathrm{C}=\mathrm{O} \rightarrow \mathrm{CO}_{2}
$$

Como puede apreciarse, el procedimiento evita cualquier referencia a las configuraciones electrónicas, pues las valencias se han enseñado, siguiendo la definición clásica, a un nivel que no necesita ese soporte explicativo. Tampoco es necesario detallar que la unión de elementos conduce a un tipo de enlace u otro. Por tanto, no hay que suponer que, aunque marcada con un trazo, la unión es necesariamente un enlace covalente (ver lo dicho en "Principios básicos" $\left.\mathrm{n}^{\circ} 4\right)$. En todo caso los alumnos podrán estudiar y aclarar más adelante las complejidades apuntadas.

\section{R4. Ámbito de la formulación}

No van a rebasarse los límites de cuatro tipos de compuestos: óxidos, hidróxidos, ácidos y sales. Los primeros se han visto en los ejemplos anteriores. Para formular los hidróxidos basta saber que están constituidos por un metal unido al grupo hidróxido (-OH). Este grupo funciona en bloque con valencia 1, ya que podemos imaginar que se deriva de la molécula $\mathrm{H}_{2} \mathrm{O}$ tras perder un $\mathrm{H}$. Conviene practicar la formulación de óxidos e hidróxidos con los ocho elementos cuyas valencias se han estudiado (Esquema 1) antes de seguir adelante.

En el caso de los ácidos oxácidos la formulación debe abandonar el camino tradicional de partir del anhídrido, "sumarle" agua (¿cuántas moléculas?) y con frecuencia "dividir" por 2. Proponemos otra vía más simple: memorizar directamente las fórmulas de los ácidos más 
importantes (oxácidos e hidrácidos). Hemos seleccionado sólo unos cuantos (cinco), que son más que suficiente para nuestros propósitos (Esquema 4). De esta manera estamos ya en disposición de deducir las fórmulas de las sales. Se sigue el procedimiento de sustituir los $\mathrm{H}$ del ácido (por cada $\mathrm{H}$, una valencia libre) por un metal, teniendo siempre en cuenta que el grupo resultante funciona como un bloque (Esquema 4, der.)

Acerca de la nomenclatura, va a utilizarse un sistema mixto. Así, la sistemática se reservará para compuestos binarios de no metales; la de Stock se empleará para óxidos metálicos e hidróxidos; y la tradicional para ácidos y sales. En este último caso la terminación del ácido determina el nombre del grupo y con él, el de la sal correspondiente. En esta, si el metal tiene valencia múltiple, se designa según el criterio de Stock con la valencia entre paréntesis.

Esquema 4

\begin{tabular}{|c|c|c|}
\hline Ácidos & $\rightarrow$ & Sales (grupo y val.) \\
\hline $\begin{array}{l}\mathrm{H}_{2} \mathrm{SO}_{4} \\
\text { sulfúrico }\end{array}$ & $\rightarrow$ & $\begin{array}{l}=\mathrm{SO}_{4} \ldots . . . \mathrm{II} \\
\text { sulfato (-ico } \rightarrow \text {-ato })\end{array}$ \\
\hline $\begin{array}{l}\mathrm{H}_{2} \mathrm{SO}_{3} \\
\text { sulfuroso }\end{array}$ & $\rightarrow$ & $\begin{array}{l}=\mathrm{SO}_{3} \ldots . . . \mathrm{II} \\
\text { sulfito } \quad \text { (-oso } \rightarrow \text {-ito) }\end{array}$ \\
\hline $\begin{array}{c}\mathrm{HCl} \\
\text { clorhídrico }\end{array}$ & $\rightarrow$ & $\begin{array}{l}-\mathrm{Cl} . . . . \mathrm{I} \\
\text { cloruro (-hídrico } \rightarrow \text {-uro) }\end{array}$ \\
\hline $\begin{array}{c}\mathrm{H}_{2} \mathrm{CO}_{3} \\
\text { carbónico }\end{array}$ & $\rightarrow$ & $\begin{array}{l}=\mathrm{CO}_{3} \ldots . . . I 1 \\
\text { carbonato }\end{array}$ \\
\hline $\begin{array}{l}\mathrm{HNO}_{3} \\
\text { nítrico }\end{array}$ & $\rightarrow$ & $\begin{array}{l}-\mathrm{NO}_{3} \ldots . . . \mathrm{I} \\
\text { nitrato }\end{array}$ \\
\hline
\end{tabular}

\section{Reflexiones y conclusiones}

La formulación química es un tema muy espinoso por sus dificultades de enseñanza y, al mismo tiempo, porque puede ser una de las principales causas de abandono de los estudios de ciencias. Por tal motivo es necesario cuidar al máximo la cuestión de su enseñanza, que, por todas las referencias, es manifiestamente mejorable.

En el presente trabajo se ha abordado el problema desde su raíz, investigando las propuestas de futuros profesores sobre la enseñanza de la formulación a niveles de $3^{\circ}-4^{\circ}$ de ESO. Los resultados obtenidos revelan un entramado de ideas y estrategias poco deseables, que, en conjunto, pueden contemplarse como modelo docente espontáneo. Este sería la consecuencia de un enfoque centrado exclusivamente en el conocimiento del contenido, CC, mientras se ignora el conocimiento didáctico del mismo, CDC (Park et al., 2011). Aunque las tendencias se han estudiado en profesores iniciales (Mellado, 1996), no son descartables entre profesores en ejercicio, pese a su mayor experiencia.

Podría alegarse que hoy día la tarea de enseñar formulación es acometida con éxito por las TICs (Muñoz, 2010). Es cierto que se produce una mejora muy sustancial si se compara un mismo diseño con y sin TIC. Pero también se constata que los programas educativos con apoyo de este recurso emplean, en general, una estrategia semejante, cuando no idéntica, a la expresada en el modelo docente espontaneo. El aumento de efectividad no se debe pues a la metodología, sino a la utilización de las TICs acopladas a pasatiempos, cuya aceptación por 
parte de los alumnos es evidentemente mejor. Nosotros, en nuestra propuesta alternativa, no hemos incidido en la forma de presentación, sino en el aspecto más profundo del problema: sus fundamentos didácticos.

Conocido entonces el esquema de enseñanza que el futuro profesor considera idóneo, y discutidos sus principales defectos, se ha diseñado la secuencia de un curso de formación inicial. Su primera etapa está dedicada a la puesta en cuestión de las concepciones manifestadas y la segunda se centra en una propuesta alternativa.

El diseño de la nueva propuesta ha promovido, además de un cambio en la metodología de enseñanza, una auténtica transposición didáctica. El resultado es que la propuesta inicial de corte disciplinar ha sido modificada y adaptada al nivel de los alumnos de ESO. La tarea se ha llevado a cabo siguiendo varias directrices. Principalmente restringir los contenidos en nivel y cantidad para lograr un aprendizaje más efectivo. La operación de formular, concebida como un establecimiento de relaciones entre elementos, avala además que pueda enseñarse con un reducido número de estos y sin necesidad del marco de la tabla periódica. Quizás esto último pueda despertar recelos e inquietudes en algún sector del profesorado, pero entiéndase bien que la propuesta alternativa es una propuesta abierta que no cierra definitivamente el aprendizaje de la formulación. Por el contrario, una vez consolidada admite nuevas incorporaciones, tanto de elementos como de compuestos (p.ej. sales ácidas). Esta característica permite encajar la propuesta dentro del ámbito de la competencia "aprender a aprender" porque, una vez construido el entramado, el alumno podrá incorporar fácilmente alguna otra sustancia o tópico a medida que vayan surgiendo.

Por otra parte, en el procedimiento de obtener las fórmulas se ha prescindido de las reglas habituales, que aparecen como arbitrarias, y se ha propuesto otra basada en el concepto de valencia. Hemos considerado este concepto como central en el proceso y hemos recurrido a la historia para asegurarnos que, siendo la valencia un concepto anterior a las teorías electrónicas del átomo y enlace (Zavaleta, 1988), se justifica poder iniciar la formulación sin dichos tópicos.

El resultado es un procedimiento austero en contenidos, a diferencia de los habituales que los movilizan en gran cantidad. Si a esto añadimos la forma razonada, no operativa, en que puede enseñarse, podrá decirse que es un esquema idóneo para abordar la formulación.

Antes de terminar debemos hacer una breve referencia a un aspecto muy a tener en cuenta como es la dimensión afectiva del aprendizaje. El impacto emocional que una determinada enseñanza puede tener sobre el alumno es un tema al cual la investigación didáctica presta gran atención en estos últimos tiempos (Zembylas, 2005; Garritz, 2009). Este aspecto juega a favor de nuestra propuesta, pues el alumno la ve asequible en nivel y contenidos y valora que gracias a sus reducidos límites sea posible abarcarla inmediatamente en su totalidad. De esta manera, el sentirse capaz de dominar un tema que tiene fama de arduo y enrevesado, le proporciona una mejora de las actitudes hacia la disciplina y un reforzamiento de su autoestima.

\section{Referencias bibliográficas}

ABELL, S.K. (2007). Research on Science Teacher Knowledge. En S.K. Abell \& N.G. Lederman (Eds.) Handbook of Research on Science Education, pp. 1105-1140. New Jersey: L. Erlbaum Ass. Inc.

ACEVEDO DÍAZ, J.A. (2009). Conocimiento didáctico del contenido para la enseñanza de la naturaleza de la ciencia (I): El marco teórico. Revista Eureka sobre Enseñanza y Divulgación de las Ciencias, 6(1), 21-46 [En línea]. 
BURGOON, J.N., HEDDLE, M.L. \& DURAN, E. (2011). Re-Examining the Similarities Between Teacher and Student Conceptions About Physical Science. Journal of Science Teacher Education 22(2), 101-114.

CARRASCOSA ALÍS, J., MARTÍNEZ TORREGROSA, J., FURIÓ MÁS, C. y GUISASOLA ARANZÁBAL, J. (2008). ¿Qué hacer en la formación inicial del profesorado de ciencias de secundaria? Revista Eureka sobre Enseñanza y Divulgación de las Ciencias, 5(2), 118-133 [En línea].

CHEVALLARD, Y. (1997). La transposición didáctica: del saber sabio al saber enseñado. Buenos Aires: Aique.

DRIVER, R. (1986). Psicología cognoscitiva y esquemas conceptuales de los alumnos. Enseñanza de las Ciencias, 4(1), 3-15.

GABEL, D. (1998). The complexity of chemistry and implications for teaching. En B.J. Fraser \& K.G. Tobin (Eds.) International handbook of science education, pp. 233-248. Boston: Kluwer.

GARRITZ, A. (2009). La afectividad en la enseñanza de la ciencia. Educación Química, 20E, 212-219.

GRIFFITHS, A.K. y PRESTON, K.R. (1992). Grade-12 students' misconceptions relating to fundamental characteristics of atoms and molecules. Journal of Research in Science Teaching, 29(6), 611-628.

HERNÁNDEZ, J. y PALACÍN, L. (1993). La formulación en el nuevo currículum de química. Comunicación, Lenguaje y Educación, 19-20, 101-107.

LÓPEZ-GAY, R. (2012). Los docentes noveles ante la preparación de las clases de ciencias. Alambique, 72, 65-74.

MEC (2007). Real Decreto 1631/2006 de enseñanzas mínimas correspondientes a la Educación Secundaria Obligatoria. BOE, 05-01-2007, 677-773.

MELLADO, V. (1996). Concepciones y prácticas de aula de profesores de ciencias, en formación inicial de Primaria y Secundaria. Enseñanza de las Ciencias, 14(3), 289-302.

MELLADO, V. y GONZÁLEZ, T. (2000). La formación inicial del profesorado de ciencias. En F. J. Perales y P. Cañal (Eds.) Didáctica de las Ciencias Experimentales (pp. 535-555). Alcoy: Marfil.

MUÑOZ CALLE, J.M. (2010). Juegos educativos. F y Q formulación. Revista Eureka sobre Enseñanza y Divulgación de las Ciencias, 7(2), 559-565 [En línea].

PARK, S., JANG, J.Y., CHEN, Y.C. \& HUNG, J. (2011). Is Pedagogical Content Knowledge (PCK) Necessary for Reformed Science Teaching?: Evidence from a Empirical Study. Research in Science Education, 41, 245-260.

ROCARD, M., CSERMELY, P., JORDE, D.; LENZEN, D., WALWERG-HENRIKSSON, H., HEMMO, V. (2008). Enseñanza de las ciencias ahora: Una nueva pedagogía para el futuro de Europa. Alambique, 55, 104-117.

ROEHRIG, G.H., KRUSE, R.A., \& KERN, A. (2007). Teacher and School Characteristics and Their Influence on Curriculum Implementation. Journal of Research in Science Teaching, 44(7), 883-907. 
SHULMAN, L.S. (1985). Paradigms and research programs in the study of teaching: A contemporary perspective. En M. C. Wittrock (Ed.) Handbook of Research on Teaching (pp. 3-36). New York: Macmillan.

SHULMAN, L. S. (1987). Knowledge and teaching: foundations of the new reform. Harvard Educational Review, 57(1), 1-22 [Traducido en línea]

SINGER, J., LOTTER, C., FELLER, R. \& GATES, H. (2011). Exploring a Model of Situated Professional Development: Impact on Classroom Practice. Journal of Science Teacher Education 22(3), 203-227.

SMITH, D.W. (2005). Valence, Covalence, Hypervalence, Oxidation State, and Coordination Number. Journal of Chemical Education, 82(8), 1202-1204.

TASKIN, V. \& BERNHOLT, S. (2012). Students' Understanding of Chemical Formulae: A review of empirical research. International Journal of Science Education, 1-29. DOI: 10.1080/09500693.2012.744492

VALCÁRCEL, M. V. y SÁNCHEZ, G. (2000). La formación del profesorado en ejercicio. En F. J. Perales, y P. Cañal (Eds.) Didáctica de las Ciencias Experimentales (pp. 557-582). Alcoy: Marfil.

VÁZQUEZ, A. y MANASSERO, M.A. (2008). El declive de las actitudes hacia la ciencia de los estudiantes: Un indicador inquietante para la educación científica. Revista Eureka sobre Enseñanza y Divulgación de las Ciencias, 5(3), 274-292 [En línea].

WOOLF, A.A. (1988). Oxidation Numbers and Their Limitations. Journal of Chemical Education, 65(1), 45-46.

ZAVALETA, D. (1988). Paradigms and plastics facts in the history of valence. Journal of Chemical Education, 65(8), 677-680.

ZEIDLER, D.L. (2002). Dancing with Maggots and Saints: Visions for Suject Matter Knowledge, Pedagogical Knowledge, and Pedagogical Content Knowledge in Science Teacher Education Reform. Journal of Science Teacher Education, 13(1), 27-42.

ZEMBYLAS, M. (2005). Three perspectives on linking the cognitive and the emotional in science learning: Conceptual change, socio-constructivism and poststructuralism. Studies in Science Education, 41(1), 91-115.

WU, H.K., KRAJCIK, J.S. y SOLOWAY, E. (2001). Promoting Understanding of Chemical Representations: Students' Use of a Visualization Tool in the Classroom. Journal of Research in Science Teaching, 38(7), 821-842. 